\title{
Is Uncertainty a Barrier or Resource to Advance Science? The Role of Uncertainty in Science and Its Implications for Science Teaching and Learning
}

\author{
Kostas Kampourakis, Kevin McCain (2019): Uncertainty: How It \\ Makes Science Advance. Oxford University Press, New York, NY. ISBN: \\ 978-0-19-0871-6, xii + 252 pages, price: $\$ 29.95$ (Hardcover), https://doi. \\ org/10.1093/oso/9780190871666.001.0001
}

\section{Ying-Chih Chen ${ }^{1}$}

Accepted: 6 May 2021

(c) This is a U.S. government work and not under copyright protection in the U.S.; foreign copyright protection may apply 2021

The COVID-19 pandemic authentically situates us in an environment of many uncertainties, pushing us to rethink the role of uncertainty in science and in public debate of several issues in our society. Throughout the pandemic, uncertainty has been inherent in a wide range of areas, from virus behaviors to immunology to health care systems. Scientists deal with uncertainty as they collect granular and disaggregated data to model the epidemiologic transition. Even as some uncertainties are resolved, more are raised, such as to what extent a recovered patient is immune from future infection, strategies to develop an effective vaccine (e.g., genetic blueprint, protein-based, or virus-based), how long the vaccine will be effective, how many doses will be needed, and the effectiveness of the vaccine against new variants of the virus. Scientists must continuously work through waves of uncertainty to develop and further their understanding of COVID-19, as with any phenomenon.

This book, "Uncertainty: How it makes science advance," was published before the COVID-19 crisis began to spread across the world in February 2020. It was written by two professors who come from two different but related fields: Dr. Kostas Kampourakis studies public understanding of the nature of science (NOS) and evolution and genetics, while Dr. Kevin McCain's expertise is in philosophy within the realm of epistemology. They eloquently highlight the critical role of uncertainty in advancing science, which can be nicely applied to the challenges and progresses we have seen during the pandemic. Importantly, this book emphasizes why we as the general public should learn how to appreciate uncertainty as a resource to advance science understanding, as scientists do. As Firestein (2012, Ignorance: How it drives science) states, "Being a scientist requires having faith in uncertainty, finding pleasure in mystery, and learning to cultivate doubt" (p. 17). The authors

Ying-Chih Chen

ychen495@asu.edu

1 Division of Teacher Preparation, Mary Lou Fulton Teachers College, Arizona State University, Tempe, AZ, USA 
organize the book into three main parts: dealing with uncertainty, uncertainties in science, and accepting uncertainty in science. In this review, I provide some of their main thoughts and my feedback and suggestions based on these three parts. Then, I provide my thoughts about the role of uncertainty in science education, building off the ideas presented in this book.

\section{Part I: Dealing with Uncertainty}

The first part of this book defines scientific knowledge, types of uncertainty, and trustiness of science. Scientific knowledge "require[s] that what you believe is true but also that you have good evidence in support of your belief" (p. 5). Based on this definition, the authors claim that establishing scientific knowledge requires two conditions: psychological and epistemic certainty. Psychological certainty refers to "how strong we believe something" (p. 6), while epistemic certainty refers to "the strength of our evidence when it comes to particular belief" (p. 37). Psychological certainty can be established without considering the sufficiency and appropriateness of evidence. In contrast, epistemic certainty can be established only based on the quality of evidence. Unfortunately, in many cases in everyday life, people become psychologically certain about a phenomenon despite their conviction being unrelated to the strength of their evidence. Therefore, psychological certainty often stops people from further inquiry. Based on this logic, epistemic certainty is more critical in science.

In general, the more psychologically certain a person is, the harder it is for that person to change and reconsider other available evidence against the original knowledge. There is often a distinction between how people of the general public versus scientists deal with something they are unsure about. People of the general public tend to pursue psychological certainty more than epistemic certainty, whereas scientists tend to pursue epistemic certainty to reduce their psychological uncertainty. However, this does not mean that scientists are always objective and have no biases because their findings are based on "evidence." As the authors point out, most published research findings do not lead to certainty, as scientists usually have different views, ways, and methods to interpret data, even if the data are the same. The authors state that "it [data] does not even speak for itself" (p. 144) and that "everything in science is a matter of interpretation" (p. 65). If this is the case, why do we still trust science?

As the authors claim, the reason is because science has a self-correcting nature. That is, scientists are "well aware of what they do not know and what they might never know" (p. 49). They accept critique and feedback to revise or correct their interpretations of raw data if there is stronger evidence suggested. This is how scientists manage epistemic and psychological uncertainties.

The authors discreetly unpack the relationship between uncertainty and scientific knowledge at an individual level, but not at a social level. They scratch the surface of social uncertainty, but do not go in depth enough. The authors do mention that scientific knowledge is established when there is a consensus within the scientific community. However, they do not go further to define and explain this idea as the social uncertainty that scientists must manage in order to establish scientific knowledge accepted by the community. This is important not only to provide a better interpretation of raw data, but also to understand and interpret peers' views, critiques, perspectives, and feedback. This standpoint resonates with Kuhn's (1962, The structure of scientific revolutions) concepts of scientific revolutions and 
paradigm shift, which claim that science can move forward because scientists solve problems accumulatively and socially based on evidence. They examine anomalies against other scientists' and their own psychological certainties. This social aspect of science is a critical method to "correct" science itself. Social uncertainty creates a motivation for scientists to provide more evidence and improve the quality of evidence to resolve epistemic disagreement. Once social uncertainty is solved, only then can scientific knowledge be established.

\section{Part II: Uncertainties in Science: Some Case Studies}

The second part of this book uses five disciplinary-based cases from climate change, vaccination, human evolution, genetic testing, and forensics to illustrate a major concept: uncertainty is inherent in science and is necessary to make science advance. In all five cases, the authors focus on epistemic uncertainty rather than other types. Across the five cases, the authors demonstrate that epistemic uncertainty has a discipline-specific nature, meaning that it is displayed in different forms in different disciplinaries. For example, the major epistemic uncertainties in climate science are inherent in the mechanisms to predict climate and the degree of human influence upon it. In the case of vaccination, uncertainty is inherent in the effectiveness of a given vaccine on different people and the risk of vaccines. In human evolution, uncertainty is inherent in that epistemic access to the past is always indirect and limited. For genetic testing, uncertainty lies in the association between a particular DNA variant and a disease, and whether a treatment will be effective. In forensic science, uncertainty is inherent in DNA profiling technologies, human errors and biases, mislabeling of samples, and misinterpretation of test results.

By examining the different forms of epistemic uncertainty inherent in the five disciplinary cases, some important features of epistemic uncertainty begin to emerge. First, there is inherent epistemic uncertainty when raw data are interpreted to shape rigorous evidence. Frequently, the authors emphasize that data do not speak for themselves. Rather, it is "all a matter of interpretation" (p. 140). Second, epistemic uncertainty is generated by the insufficiency of data. This is especially true in the cases of climate science, human evolution, and forensics. For instance, scientists' ability to develop a sophisticated understanding of human evolution and origins is limited because there is no "sufficient and complete evidence," because evidence may not exist or "because we have not yet found it" (p. 118). Third, epistemic uncertainty depends on the appropriateness of evidence. Not all data are necessary, and not all can or will be interpreted for evidence. For example, in forensics, scientists make decisions regarding which of the available data from DNA fingerprinting will be used to form evidence, and this depends on the methods they use to select data and how they measure the data's appropriateness as evidence. Fourth, epistemic uncertainty lies in the limitations of methods and technology. For example, in genetic testing, it is still not certain whether current genetic tests are accurately measuring what scientists intend to measure. Fifth, epistemic uncertainty lies in human errors and biases. For example, possibilities of accidental cross-contamination or mislabeling of samples can cause uncertainty. Regarding human bias, scientists may have conflicts of interests, or their work might be influenced by their ideological, political, religious, or other beliefs (e.g., religious perspectives on climate change), which may cause epistemic uncertainty.

The second part of the book raises a central question: If there are many forms of epistemic uncertainty inherent in science, why should we still trust science? How does science advance if uncertainty is always present? 


\section{Part III: Accepting Uncertainty in Science}

To respond to these central questions, the authors argue that "science aims at understanding, not certainty" (p. 194). That is, the goal of science is not to eliminate uncertainty, because this is not possible. Rather, the goal is to increase our understanding of the world. This echoes the ideas of Nobel Prize-winning physicist Percy Williams Bridgman, who stated that "the act of understanding is at the heart of all scientific activity" (p. 72). If we think of science as a set of certain knowledge, then we are misunderstanding the nature of science. The authors strongly emphasize that science is never certain and that "certainty is unattainable" (p. 200). They further explain that uncertainty is the fundamental nature of science for two reasons: extreme complexity of the target phenomena and human limitations.

First, we must recognize that the nature of scientific methods and explanations is fundamentally uncertain. Natural phenomena are so complex that we can never completely capture a "full" picture of them. Therefore, when scientists try to understand and explain a phenomenon, they must limit, idealize, and amplify some aspect of the phenomenon to construct a model that begins to explain it. That is, the models are idealizations and simplifications that cannot perfectly represent and fully describe every single aspect of a phenomenon, only an approximation. For example, Newton's law of universal gravitation, which describes the attraction between two bodies with a concise mathematical formula $F=G\left(M_{1} M_{2}\right) / R^{2}$, idealizes that no other force is acting upon either body. However, in a real-world situation, such an idealization cannot exist since many different forces are acting on any given body. As another example, the gas law describes the behavior of gases whose molecules are frictionless, are not subject to intermolecular attraction, and are dimensionless. Unfortunately, there are no such gases in reality. Therefore, uncertainty is already inherent in these models. We need to understand that the purpose of these models is to help us develop a better understanding of complex phenomena.

Second, we must recognize that science is a human endeavor. That is, we are always limited in our ability to explain a phenomenon due to human subjective judgment, limited information, and limited technology. A scientific explanation can "never achieve absolute certainty" (p. 176) because new evidence and better explanations will always arise to help scientists develop a better understanding. Kampourakis and McCain explain, "Science has continually advanced throughout its history because scientific understanding has increased, even if the explanations and predictions produced are uncertain" (p. 202). Because of the nature of uncertainty, scientists continue to pursue better explanations and evidence to understand natural phenomena.

In sum, the authors productively argue that science aims to improve understanding, not pursue certainty. They focus on the role of epistemic uncertainty, but not other uncertainties inherent in science. This inspires me to raise further questions: Who decide(s) what is the best explanation? Who decide(s) if the evidence is sufficient and appropriate? Obviously, it is not individual scientists per se, but rather a community of scientists who make these decisions. The authors do mention that science is a collaborative and human endeavor. I add that scientists pose different claims and explanations regarding the same issues and data. The different arguments consequently cause uncertainties at a social level. Working to resolve the social uncertainty and establish a consensus within a community is as important to science as reducing epistemic uncertainty. However, the authors do not unpack the nature of social uncertainty in this book. In the 
future, it will be useful to explore the role of social uncertainty in science based on what the authors have defined and revealed about epistemic uncertainty.

\section{How Does This Book Inspire Teaching and Learning in K-12 Science Classrooms?}

Kampourakis and McCain comprehensively discuss the inherent role of uncertainty in science and how it progresses scientific knowledge, and they suggest that "learning how to deal with uncertainty should become a central goal of our education because we face uncertainties in all aspects of our lives" (p. 28). I am inspired by the concepts and raise critical educational questions after reading the book: How can teachers appreciate and incorporate uncertainty in science classroom teaching? How can students manage uncertainty in their science learning? Research on managing uncertainty as a pedagogical resource to support student learning in science has not been adequately explored. Teachers are often not in favor of responding to the students' uncertainty and are instead eager to remove it from their pedagogical strategies and curriculum design. Students can become anxious and resist the uncertainty of open-ended problems. The prevailing orientation toward uncertainty is to see it as a barrier to overcome, and a negative experience to be avoided. Rarely do teachers or students view uncertainty as a resource to be utilized for the learning process. From my own research experience and current literature, I propose three suggestions and discuss their implications.

\subsection{Understanding the Role of Uncertainty and Learning How to Manage Uncertainty Are Essential to Scientific Literacy}

Scientific literacy has been defined in multiple ways over the past 30 years. One productive way of defining scientific literacy is to learn how to think and practice like a scientist. If we are aware of and agree with that "all scientific explanations are uncertain" (p.176), "uncertainty and awareness of that uncertainty is a driving force for scientific advancement" (p. 206), and "since [science] is uncertain, science is continually searching for new evidence" (p. 215), then understanding the role of uncertainty and learning how to manage it should be essential goals of scientific literacy. However, current schools often focus on training students to recite scientific knowledge without understating why and how the knowledge is generated and changed. Granted, not every student who walks into a science classroom wants to become a scientist. Most will enter non-science fields for their future jobs. Science courses designed for students, including non-science majors, should consider in preparing them to become scientifically literate and thoughtful citizens. It is necessary to rethink the definition of scientific literacy and the goals of science courses in schools. It would be beneficial to investigate the role of uncertainty in science and prepare students to acquire abilities to deal with uncertainty when learning science in schools. Therefore, this new definition of scientific literacy and goals pf learning science can reconceptualize and expand Norris and Phillips's (2003, How literacy in its fundamental sense is central to scientific literacy) fundamental senses of scientific literacy, in which students not only know how to interpret evidence, but also are aware of the uncertain nature of science and know how to manage the uncertainty they encounter. That is, students need to be equipped with the capacity to pursue better explanations and know how to compare different pieces of evidence in order to advance their understanding. 
Unfortunately, as Kampourakis and McCain point out, "people may favor a science that provides absolutely certainty" (p. 38) and "tend to perceive particular scientific findings as certain, completely overlooking important uncertainties inherent in the respective procedures" (p. 40). People usually misunderstand how science works and the role of uncertainty in science. I would argue that our educational goals, curricula, and pedagogy need a fundamental change. Currently, in most schools, students are used to learning and accepting scientific models and knowledge as certain. They may not understand how scientific knowledge is developed and its limitations. They may misunderstand that science is certain and absolutely correct.

People are prone to seeking out information (possibly false) that confirms their psychological certainty, allowing them to believe something (possibly false) as absolutely correct, because they were taught to believe information as fact rather than understand how actual scientific data comes to address uncertainty in science. Thus, they do not understand uncertainty's role in science, when they encounter discussions of science that mention any uncertainties (e.g., vaccine data cannot say with $100 \%$ certainty that no one will have an adverse reaction). They all of a sudden distrust science rather than understanding that uncertainty is inherent in science. To productively educate our future citizens, it is urgent to change our classroom instructions and curricula to help students realize and appreciate the role of uncertainty in science.

\subsection{Using Uncertainty as a Pedagogical Resource to Drive Students' Learning in Science}

To help students understand the role of uncertainty in science, teachers must explicitly guide students to discuss and learn it through historical cases or social-scientific issues, as well as create an authentic learning environment that immerses students in managing uncertainty as scientists do. Scientific knowledge should not be taught as a final form for students to remember for tests, but rather should be constructed, revised, and refined by students through managing their uncertainty. Therefore, to learn science is to learn how to manage uncertainty. This requires teachers to reconceptualize their curricular materials and teaching approach. For example, in the beginning of a unit, teachers may problematize a complex phenomenon that is related to students' everyday life to raise students' uncertainty. The goal of problematizing a target phenomenon is not only to elicit students' interest, but also to support them to identify potential variables, factors, and aspects that they may want to explore. This approach is very much like what scientists do in their model-creating, in which they focus on and amplify some critical factors of a phenomenon. Students can realize that the models in their lessons and in science are fundamentally uncertain and involve their assumptions, idealizations, and revisions based on evidence. They can understand that the model never will completely represent a complex phenomenon, but it can help them better understand the phenomenon.

In order to immerse students in managing uncertainty, it is necessary to engage them in communicating, arguing, and discussing their ideas, arguments, and models with each other. This instruction will help students experience social uncertainty and know how to manage it. It can help students know how other students interpret raw data as evidence, understand what alternative claims and explanations others may have, evaluate different explanations, and determine the strengths and weakness of each explanation. More importantly, students can learn how to establish mutual understanding by solving the social uncertainty within the classroom community, as scientific communities do. 


\subsection{Using Good Uncertainty to Support Productive Struggle}

It is worth noting that uncertainty may cause students' struggles, through which students develop resistance and resilience to the uncertainty. Several researchers have advocated that struggle is essential to learning. Dewey (1933, How we think: A restatement of the relations of reflective thinking to the educative process) suggests that struggle in learning pushes students to reflect on what they know and what they do not know. Piaget (1972, Intellectual evolution from adolescence to adulthood) explains that struggle results in a process of disequilibration which leads one to restructure their existing beliefs, knowledge, and concepts. The struggle is the first step in the learning process because it creates a condition for students to explicitly connect their prior knowledge to the learning tasks, problematized phenomenon, and discussed concepts.

However, not all uncertainties have the potential to cause productive struggles. Studies show that both teachers and students may develop negative perspectives and orientations of uncertainty, leading them to ignore and remove the uncertainty from their learning paths. To avoid this, teachers must distinguish between good and bad uncertainty and purposefully incorporate good uncertainty in their teaching. Good uncertainty, also called desirable uncertainty, provides opportunities for students to engage in sensemaking processes of exploring problematizing phenomena in supportive and well-structured environments. Good uncertainty may engage students in the productive struggle of the problem-solving process. Students would then be curious to explore the uncertain challenges and would become aware of their knowledge gaps. In contrast, students who experience bad uncertainty, also called undesirable uncertainty, lack clear expectations and support. Students may feel confused and may not understand the goals of learning tasks. As a result, they are not scaffolded to engage in the problem-solving processes that eventually teach them how to resolve their uncertainties.

\section{Understanding and Managing Uncertainty in a Post-truth World}

With today's rapid technology improvement and revolution, many commentators argue that we are currently living in a "post-truth world," in which misinformation, disinformation, malinformation, and fake news are pervasive in digital medias and life. People tend to pursue psychological certainty that is not matched by epistemic certainty. Kampourakis and McCain comment on this dangerous behavior as "dogmatically accepting something as true" (p. 209), not based on evidence but "belief." In the post-truth world, scientific knowledge is not sufficient to evaluate and make decisions on what is true or false information. This requires people to understand the role of uncertainty and know how to productively manage it based on evidence. This also requires our schools to not only focus on psychological uncertainty, but also acknowledge epistemic and social uncertainty. To achieve this goal, science education in K-12 schools can provide a great opportunity for students to distinguish between different types of uncertainties, learn how to productively manage uncertainty, and practice how scientific knowledge advances and is driven by uncertainty.

Publisher's Note Springer Nature remains neutral with regard to jurisdictional claims in published maps and institutional affiliations. 